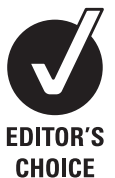

NMR Research Unit, UCL Institute of Neurology, London, UK

${ }^{2}$ Department of Neuroinflammation, UCL Institute of Neurology, London, UK

${ }^{3}$ National Hospital for Neurology and Neurosurgery, London, UK ${ }^{4}$ Department of Brain Repair and Rehabilitation, UCL Institute of Neurology, London, UK

Correspondence to Dr D Chard, Department of Neuroinflammation, UCL Institute of Neurology, Queen Square, London WC1N 3BG, UK; d.chard@ion.ucl.ac.uk

Received 9 February 2010 Revised 19 March 2010 Accepted 23 March 2010 Published Online First 2 June 2010

\title{
MRI only conversion to multiple sclerosis following a clinically isolated syndrome
}

\author{
D T Chard, ${ }^{1,2,3}$ C M Dalton, 1,2,3 J Swanton, ${ }^{1,2}$ L K Fisniku, ${ }^{1,2}$ K A Miszkiel, ${ }^{3}$ \\ A J Thompson, ${ }^{1,3,4}$ G T Plant, ${ }^{3}$ D H Miller ${ }^{1,2,3}$
}

\section{ABSTRACT}

Objectives Using current diagnostic criteria, patients who present with a clinically isolated syndrome (CIS) may develop multiple sclerosis (MS) by subsequently exhibiting dissemination in space and time on clinical (clinically definite (CD) MS) or radiological (MRI) grounds. This study investigated the frequency of radiological without clinical conversion to MS after long term follow-up as this has not previously been defined. Methods Two cohorts who underwent serial clinical and MRI studies from presentation with a CIS and who were followed-up over a mean of 6 and 20 years were investigated. The distribution and formation of lesions visible on brain MRI were assessed using the revised McDonald criteria (2005). Radiologically defined (RD) MS was determined by fulfilment of the MRI but not the CDMS criteria.

Results 105 people were followed-up for 6 years after a CIS, of whom 51\% developed CDMS, 15\% RDMS and the remainder were classified as still having had a CIS. 70 people were followed-up at 20 years, of whom $61 \%$ and $11 \%$ had developed CDMS and RDMS, respectively. Conclusion About $10-15 \%$ of CIS patients may develop MS on MRI criteria only, without further clinical events for up to two decades.

\section{INTRODUCTION}

For most patients who develop multiple sclerosis (MS), the initial clinical event is a clinically isolated syndrome (CIS) suggestive of demyelination. About two-thirds of young adults presenting with a CIS exhibit clinically silent lesions on MRI that suggest demyelination. Long term (15-20 year) follow-up studies have revealed that subsequent further clinical relapses leading to a diagnosis of clinically definite (CD) $\mathrm{MS}^{1}$ occur in $70-80 \%$ with an abnormal scan and $20-25 \%$ with normal imaging ${ }^{2}$; the majority of those who convert do so within the first 5 years. ${ }^{45}$

Since 2001, diagnostic criteria for MS allow for MRI evidence of dissemination in space and time alone to diagnose MS following a CIS, ${ }^{6}{ }^{7}$ and follow-up studies have shown that MRI conversion is often associated with developing CDMS. ${ }^{8-10}$ Although it is known that people with CIS develop clinically silent new lesions during follow-up, ${ }^{11} 12$ the frequency with which patients develop MS on MRI criteria only after prolonged follow-up has not been studied.

The work we report here sought to address the question: in the longer term, how many people could be diagnosed as having MS based on the current MRI criteria only? ${ }^{7}$

\section{METHODS}

\section{Participants}

We retrospectively interrogated data from two cohorts who underwent serial clinical and MRI studies following a CIS. The first cohort was recruited between May 1984 and July 1987 and had clinical and MRI follow-up at (means) 1, 5, 10, 14 and 20 years. $^{2}$ The second cohort was recruited between June 1995 and November 2002 and had clinical and MRI follow-up at 3 months and 1, 3 and 6 years. ${ }^{13}$ Clinical and MRI data were missing at some time points in some patients; to be included in the present study, subjects must have had both a clinical and MRI follow-up study at the final scheduled 6 year or 20 year time point.

These studies were approved by the National Hospital for Neurology and Neurosurgery and UCL Institute of Neurology Joint Research Ethics Committee. All participants gave written informed consent.

\section{MRI}

The 20 year cohort had baseline MRI and 5 year follow-up scanning undertaken using a Picker (Cleveland, USA) $0.5 \mathrm{~T}$ system, and all other scanning performed using a General Electric (Milwaukee, USA) Signa 1.5 T system. Contiguous $5 \mathrm{~mm}$ axial slices were obtained at all time points except for a minority of baseline images that were acquired with a slice thickness of $10 \mathrm{~mm}$. At baseline and 5 years, spin echo scans (repetition time $(\mathrm{TR})=2000 \mathrm{~ms}$, echo time $(\mathrm{TE})=60 \mathrm{~ms}$ ) were acquired; at 10 years, conventional spin echo scans were obtained ( $\mathrm{TR}=2000 \mathrm{~ms}, \mathrm{TE}=30 / 90 \mathrm{~ms}$ ); at 14 years, fast spin echo (FSE) sequences was acquired ( $\mathrm{TR}=2000 \mathrm{~ms}$, $\mathrm{TE}=14 / 98 \mathrm{~ms}$ ); and at 20 years an FSE sequence was obtained $(T R=2000$ $\mathrm{ms}, \mathrm{TE}=17 / 102 \mathrm{~ms}$ ).

For the 6 year cohort, all scanning was undertaken using a General Electric Signa 1.5 T system. Contiguous $3 \mathrm{~mm}$ axial slices were obtained at all time points. An FSE sequences ( $\mathrm{TR}=3200 \mathrm{~ms}$, $\mathrm{TE}=15 / 90 \mathrm{~ms}$ ) was acquired during each scanning session, and at baseline a $\mathrm{T} 1$ weighted $(\mathrm{TR}=600$ $\mathrm{ms}, \mathrm{TE}=17 \mathrm{~ms}$ ) post-gadolinium (Gd) $(0.1 \mathrm{mmol} /$ $\mathrm{kg}$ body weight) image was also obtained.

Brain T2 weighted lesion counts and volumes were determined from baseline images in both the 20 and 6 year cohorts. ${ }^{14}$ Baseline brain Gd enhancing lesion counts were obtained in the 6 year cohort.

\section{Clinical assessment}

Patients were classified into four subgroups at the last follow-up. 
1. Clinically definite (CD) MS: those people who had at least one new clinical relapse with documented new symptoms and signs, or who developed progressive neurological deficits. ${ }^{1}$

2. Radiologically defined (RD) MS: fulfilling the McDonald $\mathrm{MRI}^{7}$ criteria for dissemination in space and time, but not the CDMS criteria.

3. Evolving (E) CIS: not fulfilling CDMS or McDonald MRI criteria for MS while still accruing new MRI lesions.

4. Stable (S) CIS: with neither clinical nor MRI evidence for new disease.

Expanded Disability Status Scale (EDSS) ${ }^{15}$ scores are reported from the baseline and 6 year follow-up visits for the 6 year cohort and from the 5 and 20 year follow-up visits for the 20 year cohort (a baseline EDSS was not obtained in this group). Disease modifying treatments were prescribed only to patients with CDMS who had experienced two or more relapses in the previous 2 years.

\section{Statistical analyses}

Fisher's exact tests were used to assess group wide differences in gender ratios; all other measures were compared using Mann-Whitney U tests. Analyses were performed using SPSS 11 (SPSS Inc).

\section{RESULTS}

\section{Six year CIS group}

The 6 year CIS group were first reviewed an average of 47 days after their first clinical event (median 45, range 2-106). They were followed-up for a mean of 6.3 years (median 5.6, range 4.7-10.2 years), at which time $\sim 51 \%$ had CDMS, $\sim 15 \%$ RDMS, $\sim 12 \%$ ECIS and $\sim 20 \%$ SCIS. Comparing baseline observations between CDMS and RDMS, except for a greater proportion of males in the RDMS group $(p=0.005)$ and a trend towards the RDMS group being older at the time of their first episode $(p=0.068)$, no significant differences were detected (baseline T2 lesion volumes and numbers, Gd enhancing lesion counts and EDSS scores). Comparing CDMS with the ECIS and SCIS groups, there were significant differences in T2 lesion volume $(p<0.001)$, T2 lesion number $(p<0.001)$ and $\mathrm{Gd}$ enhancing lesion number (CDMS with ECIS, $p=0.026$ and with SCIS, $\mathrm{p}<0.001$ ) (table 1).

\section{Twenty year CIS group}

The 20 year CIS group were first reviewed an average of 63.3 days after their first clinical event (median 26, range
1-361). They were followed-up for a mean of 19.9 years (median 20, range 18.0-23.1 years), at which time $\sim 61 \%$ had CDMS, $\sim 11 \%$ had RDMS, $\sim 11 \%$ ECIS and $\sim 16 \%$ SCIS. Comparing baseline observations between CDMS and RDMS, no significant differences were detected (gender, age at first episode, baseline T2 lesion numbers and 5 year EDSS scores), although a slight trend to a lower baseline T2 lesion volume was noted ( $p=0.073$, with the caveat that T2 lesion volumes were only available in a subset of patients, as per table 2). Comparing CDMS with ECIS and SCIS, baseline T2 lesion counts were lower ( $p=0.001$ and $p<0.001$, respectively), as were T2 lesion volumes ( $p=0.002$ and $<0.001$, respectively); 5 year EDSS scores were lower in the SCIS compared with the CDMS group $(\mathrm{p}=0.048)$.

\section{DISCUSSION}

The main finding of our study was that some CIS patients (11-15\%) developed MS using current imaging but not clinical criteria after prolonged follow-up (6-20 years), so identifying a group of patients who exhibit a clinically silent disease course over a long period.

Although the length of follow-up of the two cohorts is very different, conversion to CDMS following a CIS occurs most commonly during the first 5 years. Our findings are consistent with this, showing similar proportions with CDMS in the 6 year and 20 year cohorts (51\% and $61 \%$, respectively). They also suggest that those classified as having RDMS at 5 years are more likely than not to remain so in the longer term (with $15 \%$ and $11 \%$ classified as having RDMS in the 6 year and 20 year cohorts, respectively) although some will still convert to CDMS.

With the available data, we also investigated whether there were MRI features at presentation that could help distinguish RDMS from CDMS evolution. However, like CDMS, most RDMS subjects had abnormal brain MRI at presentation with similar T2 lesion loads and, in the 6 year cohort, a similar number of gadolinium enhancing lesions. We found that the 6 year RDMS group were more often male and had a slightly older age at CIS presentation compared with the CDMS group. These clinical features tend to be over-represented in progressive forms of MS, ${ }^{16}{ }^{17}$ and their presence in the 6 year but not the 20 year RDMS group might suggest that the former cohort includes subjects who in the longer term will evolve to a secondary progressive form of disease.

It is well established that the presence of brain MRI lesions in CIS patients is associated with a relatively high likelihood of

Table 1 Clinical and MRI baseline observations, and last review EDSS scores, in the cohort followed-up over 6 years from their index clinical event

\begin{tabular}{lllll}
\hline & \multicolumn{1}{l}{ Subtype } & & & \\
\cline { 2 - 4 } & CDMS & RDMS & ECIS & SCIS \\
\hline Further clinical events & Yes & No & No & No \\
New MRI lesions & Not required & Yes & Yes & $15 / 7$ \\
Gender (F/M) & $42 / 12$ & $6 / 10$ & $8 / 5$ & $32.3(7.5)$ \\
Age at first event (years) (mean (SD)) & $33.0(7.7)$ & $36.6(6.3)$ & $34.3(7.5)$ & $0.0(0.0-0.8)$ \\
T2 lesion volume (ml) (median (range)) & $1.3(0.0-25.1)$ & $1.2(0.3-9.6)$ & $0.1(0.0-0.5)$ & $0(0-5)$ \\
T2 lesion number (median (range)) & $12(0-142)$ & $13.5(3-57)$ & $1(0-6)$ & $0(0-0)$ \\
Gd enhancing lesion number (median (range)) & $0(0-21)$ & $0(0-13)$ & $0(0-3)$ & $1.0(0.0-4.0)$ \\
First review EDSS (median (range)) & $1.0(0.0-6.0)$ & $1.0(0.0-3.0)$ & $1.0(0.0-3.0)$ \\
6 year EDSS (median (range)) & $2.0(0.0-7.5)$ & $1.0(0.0-2.0)$ & $1.0(0.0-2.0)$ & $1.0(0.0-2.5)$ \\
\hline N=105, except for baseline EDSS, where N=103 (N=53 for CDMS and N=21 for SCIS) and 6 -year EDSS scores, where N=104 \\
(N=15 for RDMS). \\
$\begin{array}{l}\text { CDMS, clinically definite multiple sclerosis; ECIS, evolving clinically isolated syndrome; EDSS, expanded disability status scale; RDMS, } \\
\text { radiologically defined multiple sclerosis; SCIS, stable clinically isolated syndrome. }\end{array}$
\end{tabular}


Table 2 Clinical and MRI baseline observations, and last review EDSS scores, in the cohort followed-up over 20 years from their index clinical event

\begin{tabular}{|c|c|c|c|c|}
\hline & \multicolumn{4}{|l|}{ Subtype } \\
\hline & CDMS & RDMS & ECIS & SCIS \\
\hline Further clinical events & Yes & No & No & No \\
\hline New MRI lesions & Not required & Yes & Yes & No \\
\hline Gender (F/M) & $31 / 12$ & $5 / 3$ & $6 / 2$ & $7 / 4$ \\
\hline Age at first event (years) (mean (SD)) & $31.5(6.4)$ & $32.6(8.5)$ & $28.5(7.6)$ & $33.3(8.4)$ \\
\hline T2 lesion volume (ml) (median (range)) & $0.8(0.0-13.7)$ & $0.0(0.0-2.3)$ & $0.0(0.0-0.4)$ & $0.0(0.0-0.0)$ \\
\hline T2 lesion number (median (range)) & $14(0-74)$ & $6(0-26)$ & $0(0-5)$ & $0(0-32)$ \\
\hline 5-year EDSS (median (range)) & $1.5(0.0-6.5)$ & $1.5(0.0-6.5)$ & $0.0(0.0-2.0)$ & $0.0(0.0-5.0)$ \\
\hline 20-year EDSS (median (range)) & $3.5(1.0-8.0)$ & $2.0(0.0-6.5)$ & $0.5(0.0-2.5)$ & $1.0(0.0-5.0)$ \\
\hline
\end{tabular}

conversion to CDMS, and disease modifying treatments such as $\beta$ interferon and glatiramer acetate delay the time to developing $\mathrm{CDMS}^{18-21}$ and are sometimes initiated in CIS patients with abnormal MRI. Our present study nevertheless highlights the fact that conversion to CDMS is not invariable and awareness of the possibility of a long term RDMS course may be useful in counselling and managing CIS patients.

In addition to the RDMS group, there was a group who accrued new lesions but did not fulfil current diagnostic criteria (ECIS). This group is likely to include subjects with a mild form of MS and some with other causes of white matter lesions, and raises the issue of the specificity of MRI findings. In this regard, the present McDonald imaging criteria for MS may beneficially reduce the chance of a false positive diagnosis of MS, albeit at the expense of diagnostic sensitivity. It has been noted that some patients who develop CDMS do not fulfil the current MRI criteria for MS, and recent work supports expanding the imaging criteria for MS in patients with a typical CIS. ${ }^{1022}{ }^{23}$ Further research to improve prediction of outcomes following a CIS is needed, and could include newer genetic, immunological and imaging measures.

There are several limitations to the study. Firstly, cognitive testing was not included in the 20 year follow-up cohort and the possibility that some of the RDMS cases developed cognitive impairment cannot be excluded. Neuropsychological testing was performed at follow-up of the 6 year cohort $^{24}$ and cognitive impairment when present was mild with no clear differences between those who developed MS and those who did not. Secondly, it is possible that some MRI lesions were not due to demyelination; particularly in the older 20 year followup cohort, some of the new white matter lesions may represent vascular disease. However, the proportion with RDMS in this group was not higher (it was actually slightly lower) than in the younger 6 year cohort, suggesting that the contribution from superadded vascular disease is probably small. Thirdly, some subjects originally recruited could not be followed-up with the required clinical and MRI evaluation at the final study time point; this was more often the case for the 20 year cohort in whom it was also not possible to scan some more disabled MS patients. Finally, there were differences between the CIS cohorts with respect to imaging protocols, frequency of scanning, type of scanner and type of CIS. Notwithstanding these factors, the two CIS cohorts exhibited many similarities (eg, age, gender and frequency of MRI abnormalities at presentation and of evolution to clinically definite MS) and the baseline clinical and imaging features of both groups are typical for CIS.
Overall, the similarity of findings in both CIS cohorts confirms that a long term RDMS course occurs, albeit less commonly than CDMS. Awareness of an RDMS course may be relevant when counselling and treating CIS patients. Further prospective follow-up studies are warranted to better characterise and understand this evolution of MS.

Acknowledgements We thank Drs Peter Brex, Kryshani Fernando, Olga Ciccarelli, Sean Morrissey, Jonathan O'Riordan and Michael Sailer for their assistance with data collection and analysis of imaging data, and the people who took part in this study.

Funding The NMR Research Unit is supported by the MS Society Great Britain and Northern Ireland, and Department of Health's NIHR Comprehensive Biomedical Research Centre at UCLH.

Competing interests $\mathrm{CMD}$ received funding from Novartis, through a grant held by the UCL Institute of Neurology, to perform MRI analysis. AJT has received honoraria for consulting services, speaking and serving on scientific advisory boards from Novartis, Eisai, Weleda/Society for Clinical Research, Hoffman La Roche, UCB Pharma, Serono Foundation, Sanofi-Aventis and the MS Society of Great Britain and Northern Ireland. He is editor-in-chief of Multiple Sclerosis for which he receives an honorarium from Sage Publications. DHM has received honoraria from UCB Pharma, Schering, Biogen Idec, GSK and Wyeth for consulting services, speaking and serving on scientific advisory boards. He has received reimbursement for work as co-chief editor of Journal of Neurology and research grant support from the MS Society of Great Britain and Northern Ireland, Wellcome Trust, Medical Research Council UK, Biogen Idec, Novartis, GlaxoSmithKline and Schering. GTP is editor-in-chief of

Neuro-Ophthalmology and the Institute of Neurology has received payments in respect of this work.

Ethics approval This study was conducted with the approval of the National Hospital for Neurology and Neurosurgery and UCL Institute of Neurology Joint Research Ethics Committee.

Provenance and peer review Not commissioned; externally peer reviewed.

\section{REFERENCES}

1. Poser CM, Paty DW, Scheinberg L, et al. New diagnostic criteria for multiple sclerosis: guidelines for research protocols. Ann Neurol 1983;13:227-31.

2. Fisniku LK, Brex PA, Altmann DR, et al. Disability and T2 MRI lesions: a 20-year follow-up of patients with relapse onset of multiple sclerosis. Brain 2008:131:808-17.

3. Optic Neuritis Study Group. Multiple sclerosis risk after optic neuritis: final optic neuritis treatment trial follow-up. Arch Neurol 2008;65:727-32.

4. Morrissey SP, Miller DH, Kendall BE, et al. The significance of brain magnetic resonance imaging abnormalities at presentation with clinically isolated syndromes suggestive of multiple sclerosis. A 5-year follow-up study. Brain 1993:116:135-46.

5. Tintoré $\mathbf{M}$, Rovira A, Río J, et al. Baseline MRI predicts future attacks and disability in clinically isolated syndromes. Neurology 2006;67:968-72.

6. McDonald WI, Compston A, Edan G, et al. Recommended diagnostic criteria for multiple sclerosis: guidelines from the International Panel on the Diagnosis of Multiple Sclerosis. Ann Neurol 2001:50:121-7.

7. Polman CH, Reingold SC, Edan G, et al. Diagnostic criteria for multiple sclerosis: 2005 revisions to the "McDonald Criteria". Ann Neurol 2005;58:840-6. 
8. Dalton CM, Brex PA, Miszkiel KA et al. Application of the new McDonald criteria to patients with clinically isolated syndromes suggestive of multiple sclerosis. Ann Neurol 2002;52:47-53.

9. Tintoré M, Rovira A, Río J, et al. New diagnostic criteria for multiple sclerosis: application in first demyelinating episode. Neurology 2003;60:27-30.

10. Swanton JK, Rovira A, Tintore M, et al. MRI criteria for multiple sclerosis in patients presenting with clinically isolated syndromes: a multicentre retrospective study. Lancet Neurol 2007;6:677-86.

11. Brex PA, Ciccarelli 0, O'Riordan Jl, et al. A longitudinal study of abnormalities on MRI and disability from multiple sclerosis. N Engl J Med 2002;346:158-64.

12. Optic Neuritis Study Group. Long-term brain magnetic resonance imaging changes after optic neuritis in patients without clinically definite multiple sclerosis. Arch Neurol 2004;61:1538-41.

13. Swanton JK, Fernando KT, Dalton CM, et al. Early MRI in optic neuritis: the risk for disability. Neurology 2009;72:542-50.

14. Grimaud J, Lai M, Thorpe J, et al. Quantification of MRI lesion load in multiple sclerosis: a comparison of three computer-assisted techniques. Magn Reson Imaging 1996;14:495-505.

15. Kurtzke JF. Rating neurologic impairment in multiple sclerosis: an expanded disability status scale (EDSS). Neurology 1983:33:1444-52.

16. Miller DH, Leary SM. Primary-progressive multiple sclerosis. Lancet Neurol 2007:6:903-12.
17. Cottrell DA, Kremenchutzky M, Rice GP, et al. The natural history of multiple sclerosis: a geographically based study. 5. The clinical features and natural history of primary progressive multiple sclerosis. Brain 1999;122:625-39.

18. Jacobs LD, Beck RW, Simon JH, et al. Intramuscular interferon beta-1a therapy initiated during a first demyelinating event in multiple sclerosis. CHAMPS Study Group. N Engl J Med 2000;343:898-904.

19. Comi G, Filippi M, Barkhof F, et al. Effect of early interferon treatment on conversion to definite multiple sclerosis: a randomised study. Lancet 2001;357:1576-82.

20. Kappos L, Polman CH, Freedman MS, et al. Treatment with interferon beta-1b delays conversion to clinically definite and McDonald MS in patients with clinically isolated syndromes. Neurology 2006;67:1242-9.

21. Comi G, Martinelli V, Rodegher $\mathrm{M}$, et al. Effect of glatiramer acetate on conversion to clinically definite multiple sclerosis in patients with clinically isolated syndrome (PreCISe study): a randomised, double-blind, placebo-controlled trial. Lancet 2009:374:1503-11.

22. Rovira A, Swanton J, Tintoré $M$, et al. A single, early magnetic resonance imaging study in the diagnosis of multiple sclerosis. Arch Neurol 2009;66:587-92.

23. Montalban X, Tintore M, Swanton JK, et al. MRI criteria for MS in patients with clinically isolated syndromes. Neurology 2010;74:427-34

24. Summers M, Swanton J, Fernando K, et al. Cognitive impairment in multiple sclerosis can be predicted by imaging early in the disease. J Neurol Neurosurg Psychiatry 2008:79:955-8. 